\title{
Incorporation of perfluorohexyl-functionalised thiophenes into oligofluorene-truxenes: synthesis and physical properties
}

Neil Thomson, Alexander L. Kanibolotsky, Joseph Cameron, Tell Tuttle, Neil J. Findlay and Peter J. Skabara*

Open Access

\author{
Full Research Paper \\ Address: \\ WestCHEM, Department of Pure and Applied Chemistry, University of \\ Strathclyde, 295 Cathedral Street, Glasgow, G1 1XL, U.K. \\ Email: \\ Peter J. Skabara* - peter.skabara@strath.ac.uk \\ * Corresponding author \\ Keywords: \\ oligomers; perfluorinated-materials; star-shaped molecules; synthesis; \\ truxene
}

\author{
Beilstein J. Org. Chem. 2013, 9, 1243-1251. \\ doi:10.3762/bjoc.9.141 \\ Received: 27 February 2013 \\ Accepted: 12 June 2013 \\ Published: 27 June 2013 \\ Associate Editor: D. O'Hagan \\ () 2013 Thomson et al; licensee Beilstein-Institut. \\ License and terms: see end of document.
}

\begin{abstract}
Oligofluorene-functionalised truxenes containing perfluorohexylthiophene units at the terminal positions on the arms were synthesised, and their optical and electrochemical properties were investigated to determine the effect that the perfluorohexylthiophene unit has on the HOMO and LUMO properties of the oligomers. By synthesising a molecule with longer oligofluorene arms the effects of the perfluorohexylthiophene unit on larger oligomers was explored. The effect of steric hindrance from the perfluorohexyl chain was also evaluated by altering the position of the chain on the thiophene moiety.
\end{abstract}

\section{Introduction}

Star-shaped oligomers share a common central unit with multiple arms branching from the core [1]. The morphology and electronic properties of these oligomers often vary from the linear versions of the arms alone.

10,15-Dihydro-5 $H$-diindeno[1,2- $\left.a ; 1^{\prime}, 2^{\prime}-c\right]$ fluorene, also known as truxene (Figure 1), is a heptacyclic polyarene with $C_{3}$ symmetry that can be envisaged as three fluorenes that are superimposed through a common central benzene ring $[2,3]$.
Truxene has been used as a starting compound, or a core unit, for larger star-shaped polyarenes such as fullerene fragments [4] and liquid-crystalline compounds [5]. Positions C5, C10 and C15 can be functionalised with a range of substituents, commonly saturated alkyl chains, which can enhance the solubility and processability as well as reduce intermolecular $\pi-\pi$ stacking [6], but there are also examples of unsaturated alkyl [7] and phenyl [8] substituents at these positions. Cross-coupling reactions at the $\mathrm{C} 2, \mathrm{C} 7$ and $\mathrm{C} 12$ positions is the usual method of 


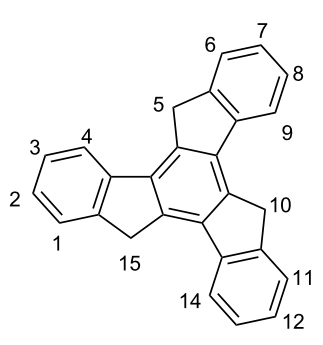

13

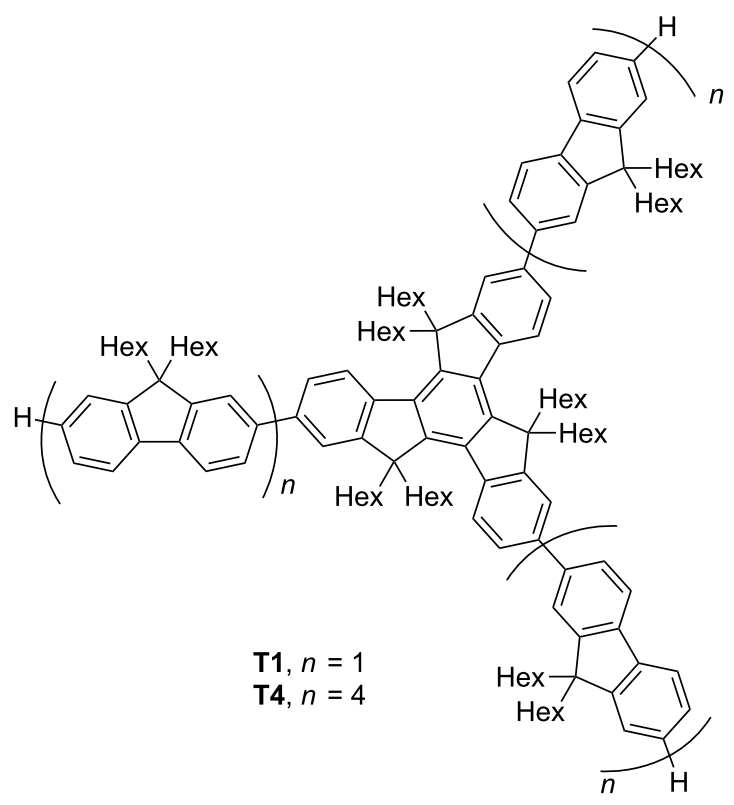

Figure 1: Labelled truxene and compounds T1 and T4.

attaching "arms" to the core to form the star-shaped oligomers, such as T1 and T4 (Figure 1), extending the conjugation length and altering the photophysical properties of the resulting oligomer. There are also less common examples of functionalisation at the $\mathrm{C} 3, \mathrm{C} 8$ and $\mathrm{C} 13$ positions [9].

Alkyl substituents attached to hetero- and carbocyles, which make up the conjugated backbone of oligomers and polymers, can also affect the properties of the material [10]. Alkyl chains or other functional groups can donate or withdraw electron density from the polymer chain, for example by inductive effects, altering the HOMO-LUMO levels and subsequent band gap in the process. One type of substituent that can be used is a perfluoroalkyl chain, where the high percentage of fluorine present gives rise to a large electron-withdrawing effect [11]. However, perfluoroalkyl halides are not suitable alkylating reagents for $\mathrm{S}_{\mathrm{N}} 2$ type reactions, which makes the attachment of these types of chains to aromatic cyclic compounds difficult without the use of a spacer group, such as an ethylene chain, which can lessen the inductive effect [12]. Due to the widespread industrial use of polythiophenes, such as P3HT, analogous poly(perfluoroalkyl)thiophenes have received some interest due to their ability to affect certain properties of the polymer, such as the electrochemistry and stability, as well as the improved chemical resistance and thermal stability that replacing alkyl hydrogen atoms with fluorine atoms affords [13].

Our group has previously published work on oligofluorenefunctionalised truxenes [14], and it was in our interest to observe changes in the optical and electrochemical properties by introducing perfluorohexylthiophene units at the terminal positions of the oligofluorene arms. Planarity of the backbone is an important concept in conjugated organic oligomers and polymers, as any twist in the dihedral angle between aromatic repeat units can lessen the p-orbital overlap, breaking the effective conjugation length [15]. This planarity can be perturbed by the steric effects of substituents attached to the hetero- or carbocycles in the conjugated backbone. In order to observe if the position of the perfluorohexyl chains would have an effect, two molecules that are structural isomers were synthesised and studied to compare the optical and electrochemical properties. Furthermore, a significantly larger molecule was synthesised to observe how a molecule with longer conjugated arms would also be affected by the perfluorohexylthiophene units.

\section{Results and Discussion}

The synthesis of the oligofluorene-functionalised truxenes is outlined in Schemes 1-4. 3-Perfluorohexylthiophene (3) was synthesised from 3-bromothiophene (1) by initial halogen exchange, using the method described by Buchwald et al. [16], to afford 3-iodothiophene (2), which then underwent a coppermediated coupling reaction with perfluorohexyl iodide (Scheme 1). Lithiation of $\mathbf{3}$ and treatment with perfluorohexyl iodide gave the product 2-iodo-3-perfluorohexylthiophene (4). The regioselectivity of this reaction was poor, and the product was obtained as a mixture with its isomer, 2-iodo-4-perfluorohexylthiophene, which was separated by column chromatography. Compound $\mathbf{4}$ then underwent Suzuki-Miyaura coupling with compound 5, which was synthesised according to our 


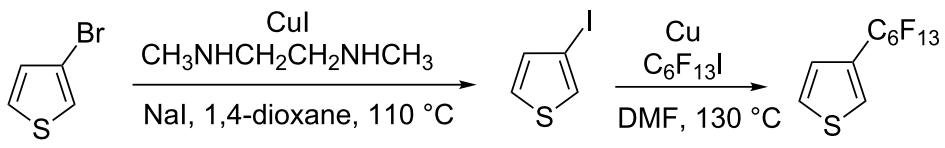

2, $75 \%$<smiles>C[SiH2]c1ccc2c(c1)C(C)(C)c1cc(Br)ccc1-2</smiles><smiles>[R]C1([14CH3])c2cc([SiH2]C)ccc2-c2ccc(-c3sccc3C(F)(F)F)cc21</smiles>

6, $80 \%$

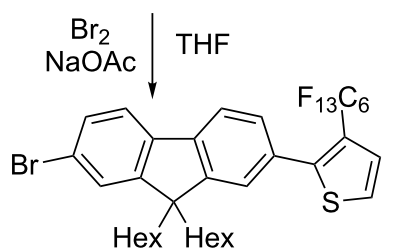

7, $82 \%$
$\mathrm{Ba}(\mathrm{OH})_{2}$

$\mathrm{THF}, 80^{\circ} \mathrm{C}$

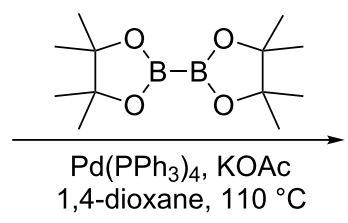

$\mathrm{Pd}\left(\mathrm{PPh}_{3}\right)_{4}, \mathrm{KOAc}^{\circ}, 4$-dioxane, $110^{\circ} \mathrm{C}$
3, $64 \%$

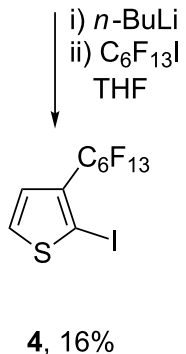

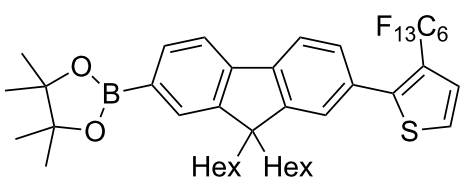

$8,46 \%$

Scheme 1: Synthesis of the thiophene-fluorene arm for the 3-isomer.

previously published method [17], and the product obtained, 6, The synthetic route to 2-iodo-4-perfluorohexylthiophene (13) was deprotected by bromination to give compound 7 and then (Scheme 2) began with treatment of 3-bromothiophene (1) with reacted with bis(pinacolato)diboron to afford boronic ester $\mathbf{8}$. lithium bis(trimethylsilyl)amide and chlorotrimethylsilane to<smiles>Brc1ccsc1</smiles>

1 i) $\operatorname{LiHMDS}$
$\stackrel{\text { ii) } \mathrm{TMSCl}}{\mathrm{THF}}$

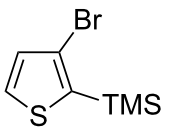

9, $92 \%$

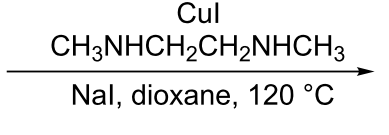$$
\text { ( }
$$<smiles>O=S(=O)(O)c1sccc1I</smiles>

$10,43 \%$ with residual 9<smiles>CC(C)c1ccsc1[AsH3]</smiles>

11, $28 \%$<smiles>[Y][C@]1([14CH3])c2cc([SiH2])ccc2-c2ccc(-c3cc(C(C)(F)F)cs3)cc21</smiles>

$14,55 \%$

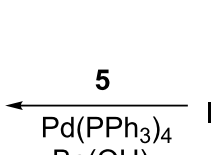
$\mathrm{Ba}(\mathrm{OH})_{2}$ THF<smiles>FC(F)(F)c1csc(I)c1</smiles>

$13,83 \%$

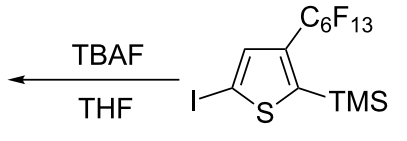

$12,87 \%$

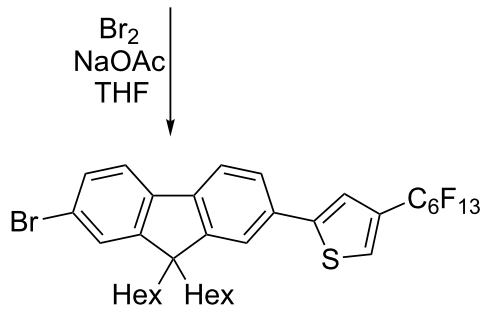

$15,91 \%$

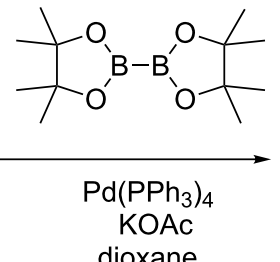

dioxane<smiles>[Y]C1([R4])c2cc(B3OC(C)(C)C(C)(C)O3)ccc2-c2ccc(-c3cc(C(F)(F)F)cs3)cc21</smiles>

$16,29 \%$ 
afford 2-trimethylsilyl-3-bromothiophene (9). However, although the conditions were unchanged, the halogen exchange reaction used to afford $\mathbf{1 0}$ was poorer yielding than that which produced 2. This was due to incomplete conversion from the bromide to the iodide. The inability to separate $\mathbf{9}$ and $\mathbf{1 0}$ meant that this mixture was not further purified, and the mixture was taken forward to the next stage of the synthesis. The subsequent copper-mediated coupling reaction to afford $\mathbf{1 1}$ was also poor-yielding and a mixture of 9,10 and 11 was obtained. Compound 11 was obtained pure by a fluorinous extraction process with perfluorohexanes from a 5\% water/ethanol solution. Lithiation and treatment with perfluorohexyliodide afforded $\mathbf{1 2}$, which was deprotected with tetrabutylammonium fluoride to afford 13. The boronic ester $\mathbf{1 6}$ was then synthesised by the same steps applied to obtain compound $\mathbf{8}$ (see Scheme 1).

T1- ${ }^{3}$ FTh and T1-4 FTh were then synthesised by Suzuki coupling of tribromohexahexyltruxene $\mathbf{1 7}$, which was synthesised according to our previously published method [14], and the relevant boronic ester, $\mathbf{8}$ or $\mathbf{1 6}$ (Scheme 3).

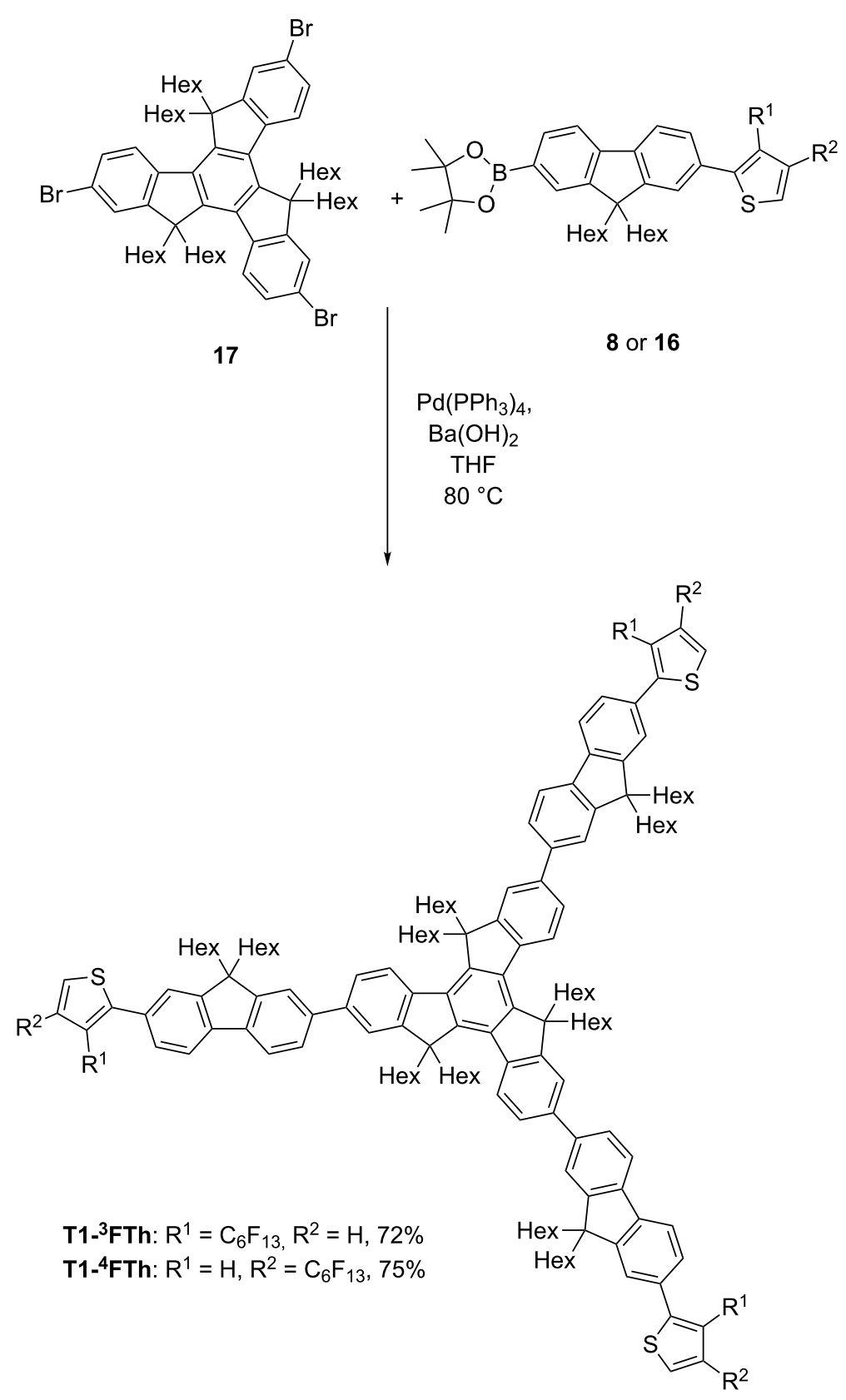

Scheme 3: Coupling of arms to the truxene core. 
T4- ${ }^{4}$ FTh (Scheme 4) was synthesised by coupling compound 16 with T3Br. The latter compound was obtained by our previously published method [17].

The absorption and emission spectra of the oligomers in solution (dichloromethane), shown in Figure 2, all exhibit strong $\pi-\pi^{*}$ transitions. The maximum absorption band was observed at $349 \mathrm{~nm}$ for T1- ${ }^{\mathbf{3}} \mathbf{F T h}, 362 \mathrm{~nm}$ for $\mathbf{T 1}-{ }^{\mathbf{4}} \mathbf{F T h}$, and $380 \mathrm{~nm}$ for T4- ${ }^{4} \mathbf{F T h}$. The maximum wavelength of emission was observed at $412 \mathrm{~nm}$ for $\mathbf{T} 1{ }^{\mathbf{3}} \mathbf{F T h}, 402 \mathrm{~nm}$ for T1- ${ }^{\mathbf{4}} \mathbf{F T h}$, and $421 \mathrm{~nm}$ for T4- ${ }^{4} \mathbf{F T h}$. In comparison to the corresponding spectra of $\mathbf{T} 1$ and T4 (Table 1), the introduction of perfluorohexylthiophene units

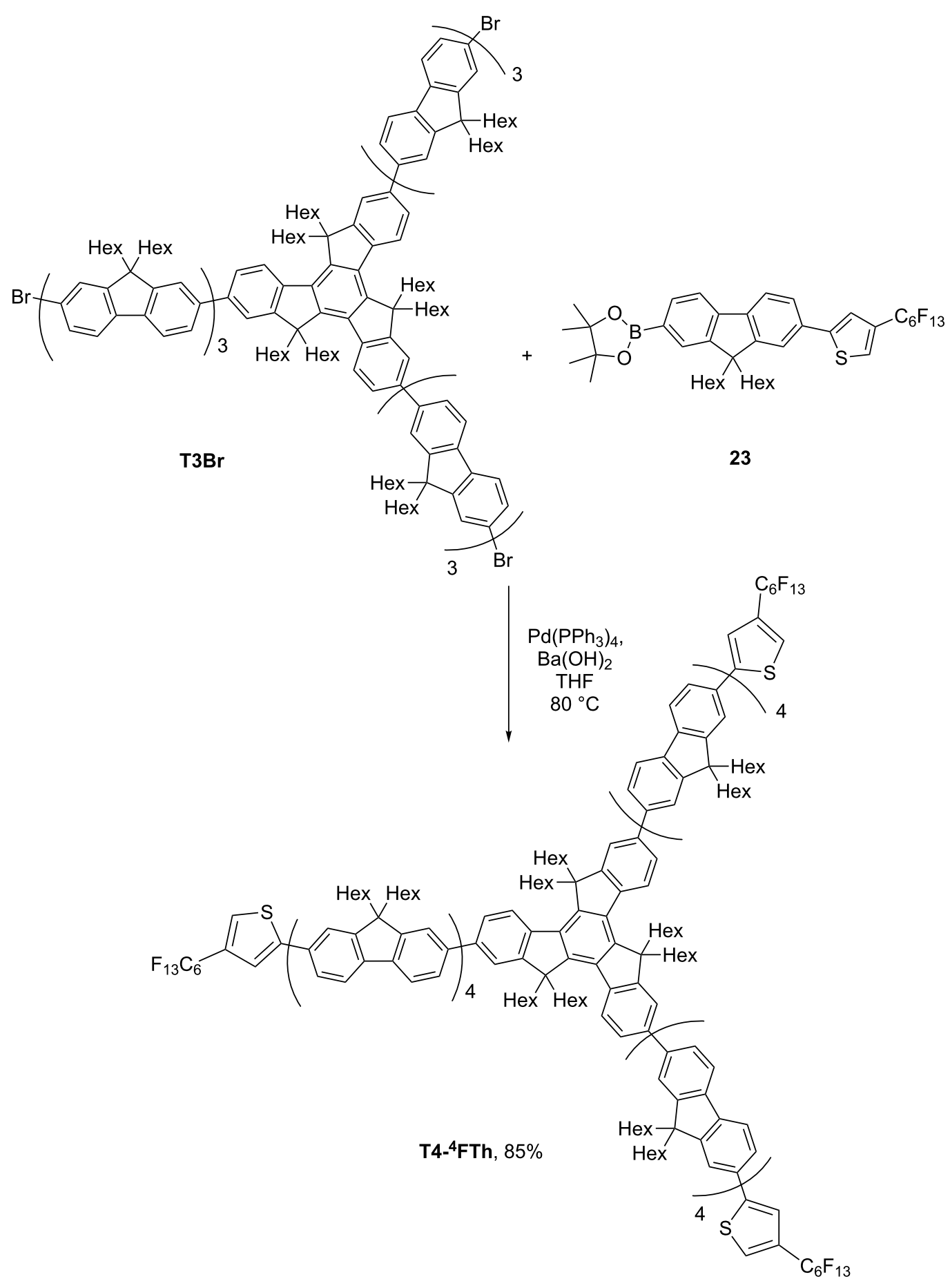




\begin{tabular}{|c|c|c|c|c|c|c|}
\hline compound & $\begin{array}{c}\lambda_{\max }(\mathrm{nm}) \text { absorption } \\
\left(\mathrm{CH}_{2} \mathrm{Cl}_{2}\right)\end{array}$ & $\begin{array}{l}\text { onset } \\
(\mathrm{nm})\end{array}$ & $\left(\mathrm{mM}^{-1} \mathrm{~cm}^{-1}\right)$ & $\begin{array}{c}\text { HOMO_LUMO gap } \\
(\mathrm{eV})\end{array}$ & $\begin{array}{c}\lambda_{\max }(\mathrm{nm}) \text { emission } \\
\left(\mathrm{CH}_{2} \mathrm{Cl}_{2}\right)\end{array}$ & $\begin{array}{c}\Phi_{\mathrm{PL}} \\
\left(\mathrm{CH}_{2} \mathrm{Cl}_{2}\right)\end{array}$ \\
\hline T1-35Th & 349 & 387 & 306 & 3.20 & 412 & - \\
\hline T1-4FTh & 362 & 397 & 323 & 3.12 & 402,418 & 0.86 \\
\hline T4-4FTh & 380 & 409 & 385 & 3.03 & 421, 440sh & 0.93 \\
\hline $\mathbf{T 1}^{\mathrm{a}}$ & 343 & - & - & 3.29 & 375 sh, 396, 416sh & - \\
\hline $\mathrm{T}^{\mathrm{a}}$ & 374 & - & - & 3.05 & $411,436,460$ sh & - \\
\hline
\end{tabular}

aValues obtained from [14].

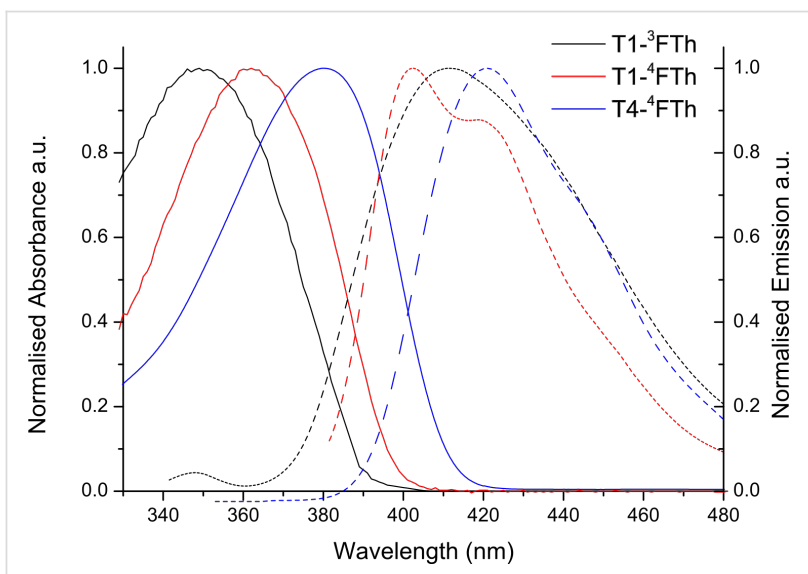

Figure 2: Normalised absorbance (solid) and emission (dashed) of materials in solution (dichloromethane). emission spectrum of T4- ${ }^{4}$ FTh also reveals the vibronic structure, although it is less pronounced, with a shoulder at $440 \mathrm{~nm}$. The Stokes shifts for T1- ${ }^{\mathbf{4}}$ FTh and T4- ${ }^{\mathbf{4}}$ FTh are 40 and $41 \mathrm{~nm}$ respectively; however, the shift for $\mathbf{T} 1 \mathbf{-}^{\mathbf{3}} \mathbf{F T h}$ is larger, at around $63 \mathrm{~nm}$. This is an indication that the excited state of T1- ${ }^{3} \mathbf{F T h}$ undergoes a greater conformational change before emission than that of $\mathbf{T 1}{ }^{4} \mathbf{F T h}$, which is further evidence of the greater planarity in the T1- ${ }^{\mathbf{4}} \mathbf{F T h}$ ground state compared to that of its isomer. The optical HOMO-LUMO gaps were estimated from the onset of absorption and were recorded as 3.20, 3.12 and $3.03 \mathrm{eV}$ for T1- ${ }^{\mathbf{3}} \mathbf{F T h}, \mathbf{T 1 -}{ }^{\mathbf{4}} \mathbf{F T h}$ and $\mathbf{T 4} \mathbf{-}^{\mathbf{4}} \mathbf{F T h}$, respectively. The HOMO-LUMO gap for T1- ${ }^{\mathbf{3}} \mathbf{F T h}$ and $\mathbf{T 1 -}{ }^{\mathbf{4}} \mathbf{F T h}$ is slightly narrower than that for T1; however, there is not much difference between the HOMO-LUMO gap of T4- ${ }^{4}$ FTh and that of T4.

The results for the cyclic voltammetry of the oligomers are given in Table 2 (see Supporting Information File 1, Figures S1-S6 for the voltammograms). T1- ${ }^{\mathbf{3}} \mathbf{F T h}$ showed one irreversible oxidation peak at $+1.09 \mathrm{~V}$, and a reduction peak was found at $-2.22 \mathrm{~V}$. From the onset of the oxidation and reduction peaks the HOMO level was estimated to be $-5.72 \mathrm{eV}$ and the LUMO $-2.74 \mathrm{eV}$ with reference to ferrocene, which was to the terminal positions on the arms leads to a red shift for both absorption and emission. The red shift within the ${ }^{\mathbf{4}} \mathbf{F T h}$-substituted series (T1-4 FTh and T4-4 FTh) increases with increasing chain length. The emission spectrum for $\mathbf{T 1}{ }^{\mathbf{4}} \mathbf{F T h}$ also shows a pronounced vibronic feature at $418 \mathrm{~nm}$, which is an indication of the planarity of the molecular ground state in comparison to that of $\mathbf{T} 1{ }^{-3} \mathbf{F T h}$, where no vibronic splitting is observed. The

Table 2: Electrochemical data for TX-XFTh and TX.

\begin{tabular}{|c|c|c|c|c|c|}
\hline compound & $\begin{array}{c}E_{\mathrm{ox}}(\mathrm{V}) \text { peaks } \\
E_{\mathrm{Pa}} / E_{\mathrm{Pc}} \text { or } E_{\mathrm{Pa}}{ }^{a}\end{array}$ & HOMO & $\begin{array}{c}E_{\text {red }}(\mathrm{V}) \text { peaks } \\
E_{\mathrm{P}}\end{array}$ & LUMO & $\begin{array}{c}\text { HOMO-LUMO gap } \\
(\mathrm{eV})\end{array}$ \\
\hline T1- ${ }^{3} \mathrm{FTh}$ & +1.09 & -5.72 & -2.22 & -2.74 & 2.98 \\
\hline T1-4 FTh & $\begin{array}{c}+0.86 / 0.79 \\
+1.03 / 0.96 \\
+1.26^{b} \\
+1.44^{b}\end{array}$ & -5.55 & -2.84 & -2.39 & 3.16 \\
\hline T4-4FTh & $\begin{array}{l}+0.91 /+0.82^{\mathrm{C}} \\
+1.11^{\mathrm{C}}\end{array}$ & -5.53 & -2.59 & -2.35 & 3.18 \\
\hline$T 1^{d}$ & - & -5.6 & - & -2.2 & 3.4 \\
\hline$T 4^{d}$ & - & -5.5 & - & -2.3 & 3.2 \\
\hline
\end{tabular}

aNo cathodic peak is reported where the wave is irreversible. ${ }^{b}$ Peak exists as a shoulder. ${ }^{c}$ Two unresolved single-electron peaks. ${ }^{d} V a l u e s$ obtained from [14]. 
used as an internal standard and has a HOMO of $-4.8 \mathrm{eV}$. In comparison to the HOMO and LUMO values for T1 [14] (-5.6 and $-2.2 \mathrm{eV}$ ) there is a decrease in the HOMO and a large decrease in the LUMO, with a reduction in the HOMO-LUMO gap. For T1-4 ${ }^{\mathbf{4} T h}$, quasi-reversible oxidation peaks were found at +0.86 and $+1.03 \mathrm{~V}$, while irreversible peaks were found as +1.26 and $+1.44 \mathrm{~V}$. A reduction peak was observed at $-2.84 \mathrm{~V}$. From the onset of the oxidation and reduction peaks the HOMO level was estimated to be $-5.55 \mathrm{eV}$, and the LUMO $-2.39 \mathrm{eV}$. Comparing these values to that of $\mathbf{T 1}$, the HOMO is essentially the same with the LUMO decreased, resulting in a narrower electrochemical band gap. A similar effect is observed for T4- ${ }^{\mathbf{4}} \mathbf{F T h}$ in comparison with T4 [14], where the HOMO is $-5.53 \mathrm{eV}$ compared to $-5.5 \mathrm{eV}$ for $\mathbf{T 4}$ and the LUMO is lower ( $-2.35 \mathrm{eV}$ for $\mathbf{T} 4{ }^{\mathbf{4}} \mathbf{F T h}$ and $-2.3 \mathrm{eV}$ for T4). The electrochemical HOMO-LUMO gaps for T1- ${ }^{3}$ FTh, T1-4FTh and T4- ${ }^{4}$ FTh were close to those derived from the absorption spectra, (within ca. $0.2 \mathrm{eV}$ ), which is in line with the differences observed for T1 and T4.
In order to rationalise the difference in optical and structural properties between the two isomers, DFT calculations were performed on the smaller truxenes. The T1- ${ }^{3}$ FTh and T1- ${ }^{4}$ FTh structures were optimised in the gas phase by using the BP86-D [18-20] functional with the def2-TZVP [21] basis set implemented in TURBOMOLE 6.3.1 [22]. The optimisations were carried out by using the RI-J approximation [23]. The hexyl chains on the fluorene units were shortened to methyl groups in the model in order to decrease the computational cost of the simulation. The main structural difference observed is the degree of twisting between the fluorene and thiophene in the two structures. The average dihedral angle in $\mathbf{T 1}-^{3} \mathbf{F T h}$ is $40.9^{\circ}$, whilst there is a much smaller twist of $6.4^{\circ}$ in T1-4FTh. The frontier orbitals of the isomers were also compared and are shown below (Figure 3 and Figure 4). The HOMO-1 and HOMO for both molecules $\left(\triangle E\left[\mathbf{T} 1 \mathbf{-}^{\mathbf{3}} \mathbf{F T h}\right]=0.0054 \mathrm{eV}\right.$ and $\left.\Delta E\left[\mathbf{T 1}{ }^{4} \mathbf{F T h}\right]=0.0030 \mathrm{eV}\right)$ are essentially degenerate and show a splitting due to a slight break in symmetry. This is also the case for the LUMO and LUMO+1 of each isomer
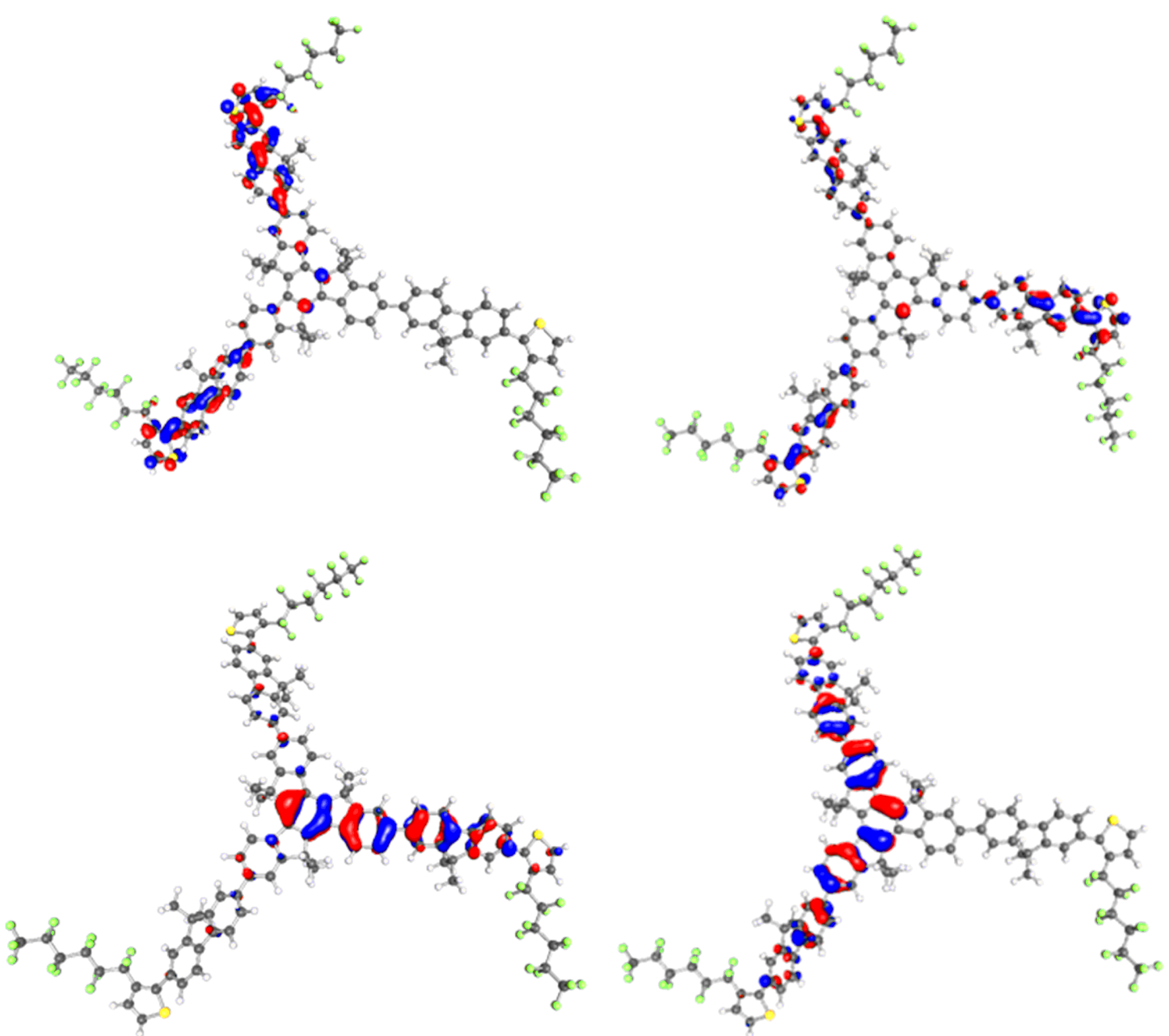

Figure 3: HOMO-1 (bottom, left), HOMO (bottom, right), LUMO (top, left) and LUMO+1 (top, right) of T1-35Th. 

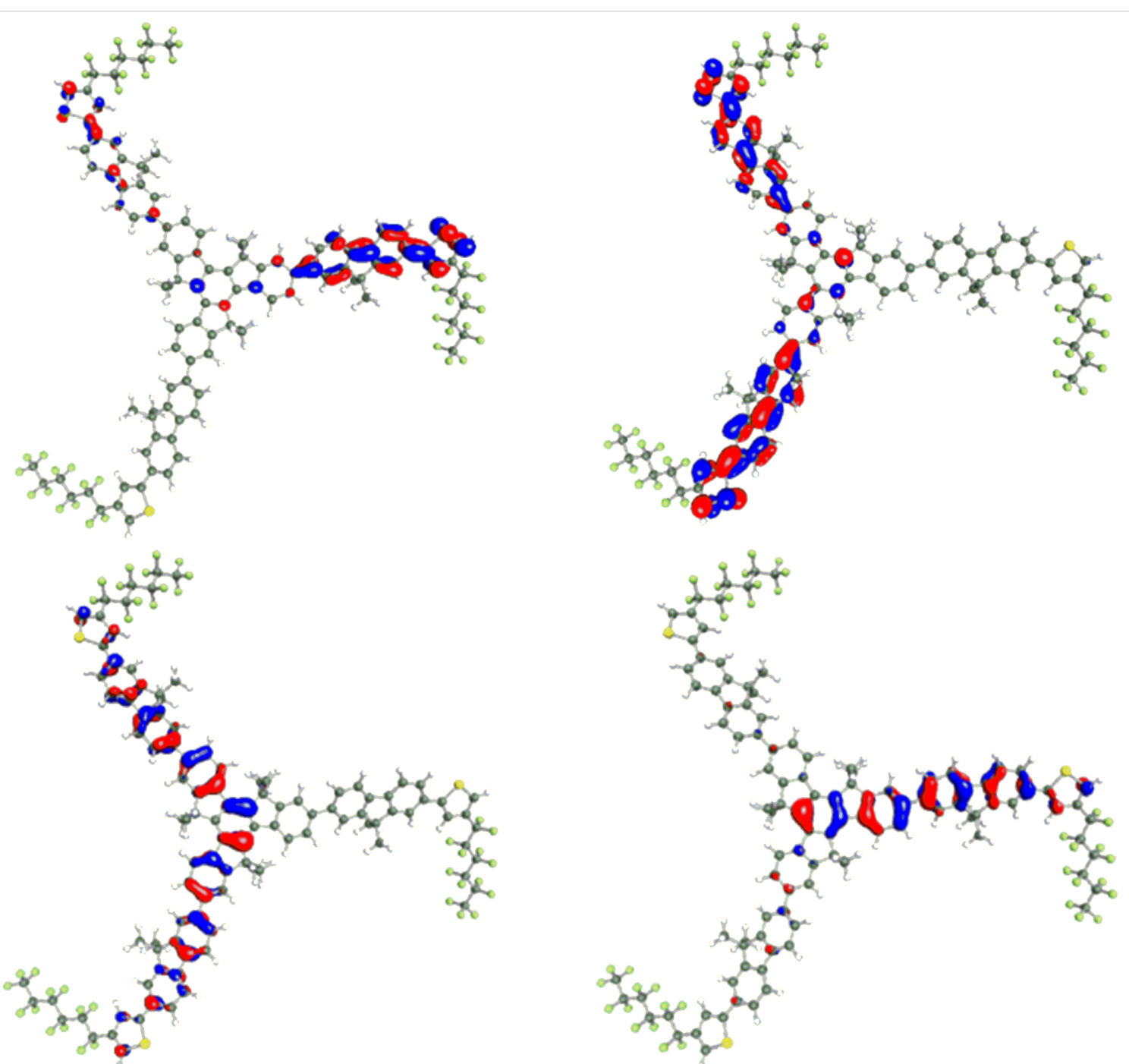

Figure 4: HOMO-1 (bottom, left), HOMO (bottom, right), LUMO (top, left) and LUMO+1 (top, right) of T1-4FTh.

$\left(\Delta E\left[\mathbf{T} 1-{ }^{3} \mathbf{F T h}\right]=0.013 \mathrm{eV}\right.$ and $\left.\Delta E\left[\mathbf{T} 1-{ }^{4} \mathbf{F T h}\right]=0.0047 \mathrm{eV}\right)$. The HOMO-1 and HOMO of the compounds show there to be a larger contribution of the thiophene unit to the conjugation of the molecule in T1-4 FTh compared to T1- ${ }^{\mathbf{3}} \mathbf{F T h}$. This reaffirms that the thiophene unit of T1-3FTh is too twisted to contribute significantly to the conjugation of the molecule.

\section{Conclusion}

In conclusion we have presented three new star-shaped oligomers, namely T1- ${ }^{3}$ FTh, T1- ${ }^{4}$ FTh, and T4- ${ }^{\mathbf{4}} \mathbf{F T h}$. For the smaller molecules, the presence of the powerfully electronwithdrawing perfluorohexyl chain within the molecules leads to a more stabilised LUMO, in comparison to the hydrogen-terminated molecule T1. The optical and electrochemical properties of the molecules with shorter oligofluorene arms are altered to a much greater extent than those of the molecules containing longer oligofluorene arms, where HOMO and LUMO levels as well as the optical HOMO-LUMO gaps remain essentially the same for the substituted and the parent systems. The electrochemical properties of T1-3 ${ }^{3} \mathbf{F T h}$ are more affected by the presence of the perfluoroalkyl thiophene substituent than those of T1- ${ }^{4}$ FTh.

\section{Supporting Information}

\section{Supporting Information File 1}

Experimental procedures and cyclic voltammetry details.

[http://www.beilstein-journals.org/bjoc/content/

supplementary/1860-5397-9-141-S1.pdf]

\section{Acknowledgements}

We would like to thank the EPSRC and Cambridge Display Technology for funding N.T. 


\section{References}

1. Kanibolotsky, A. L.; Perepichka, I. F.; Skabara, P. J. Chem. Soc. Rev. 2010, 39, 2695-2728. doi:10.1039/b918154g

2. Pei, J.; Wang, J.-L.; Cao, X.-Y.; Zhou, X.-H.; Zhang, W.-B. J. Am. Chem. Soc. 2003, 125, 9944-9945. doi:10.1021/ja0361650

3. Gómez-Lor, B.; de Frutos, Ó.; Ceballos, P. A.; Granier, T.; Echavarren, A. M. Eur. J. Org. Chem. 2001, 2107-2114. doi:10.1002/1099-0690(200106)2001:11<2107::AID-EJOC2107>3.0.C O;2-F

4. Gómez-Lor, B.; González-Cantalapiedra, E.; Ruiz, M.; de Frutos, Ó.; Cárdenas, D. J.; Santos, A.; Echavarren, A. M. Chem.-Eur. J. 2004, 10, 2601-2608. doi:10.1002/chem.200306023

5. Zhao, K.-Q.; Chen, C.; Monobe, H.; Hu, P.; Wang, B.-Q.; Shimizu, Y. Chem. Commun. 2011, 47, 6290-6292. doi:10.1039/c1cc10299k

6. Tseng, K.-P.; Kao, M.-T.; Tsai, T. W. T.; Hsu, C.-H.; Chan, J. C. C.; Shyue, J.-J.; Sun, S.-S.; Wong, K.-T. Chem. Commun. 2012, 48, 3515-3517. doi:10.1039/c2cc00099g

7. González-Cantalapiedra, E.; Ruiz, M.; Gómez-Lor, B.; Alonso, B.; García-Cuadrado, D.; Cárdenas, D. J.; Echavarren, A. M. Eur. J. Org. Chem. 2005, 4127-4140. doi:10.1002/ejoc.200500260

8. Kao, M.-T.; Chen, J.-H.; Chu, Y.-Y.; Tseng, K.-P.; Hsu, C.-H.; Wong, K.-T.; Chang, C.-W.; Hsu, C.-P.; Liu, Y.-H. Org. Lett. 2011, 13, 1714-1717. doi:10.1021/ol200227w

9. Lai, W.-Y.; He, Q.-Y.; Ma, Z.; Huang, W. Chem. Lett. 2009, 38, 286-287. doi:10.1246/cl.2009.286

10. Casado, J.; Pappenfus, T. M.; Miller, L. L.; Mann, K. R.; Ortí, E.; Viruela, P. M.; Pou-Amérigo, R.; Hernández, V.; López Navarrete, J. T. J. Am. Chem. Soc. 2003, 125, 2524-2534. doi:10.1021/ja027835p

11. Lee, J.-K.; Fong, H. H.; Zakhidov, A. A.; McCluskey, G. E.;

Taylor, P. G.; Santiago-Berrios, M.; Abruña, H. D.; Holmes, A. B. Malliaras, G. G.; Ober, C. K. Macromolecules 2010, 43, 1195-1198. doi:10.1021/ma902179s

12. Facchetti, A.; Yoon, M.-H.; Stern, C. L.; Hutchison, G. R.; Ratner, M. A.; Marks, T. J. J. Am. Chem. Soc. 2004, 126, 13480-13501. doi:10.1021/ja048988a

13. Endo, T.; Takeoka, Y.; Rikukawa, M.; Sanui, K. Synth. Met. 2003, 135-136, 333-334. doi:10.1016/S0379-6779(02)00537-4

14. Kanibolotsky, A. L.; Berridge, R.; Skabara, P. J.; Perepichka, I. F.; Bradley, D. D. C.; Koeberg, M. J. Am. Chem. Soc. 2004, 126, 13695-13702. doi:10.1021/ja039228n

15. Tsao, H. N.; Müllen, K. Chem. Soc. Rev. 2010, 39, 2372-2386. doi:10.1039/b918151m

16. Klapars, A.; Buchwald, S. L. J. Am. Chem. Soc. 2002, 124, 14844-14845. doi:10.1021/ja028865v

17. Belton, C. R.; Kanibolotsky, A. L.; Kirkpatrick, J.; Orofino, C.; Elmasly, S. E. T.; Stavrinou, P. N.; Skabara, P. J.; Bradley, D. D. C Adv. Funct. Mater. 2013, 23, 2792-2804. doi:10.1002/adfm.201202644

18. Becke, A. D. Phys. Rev. A 1988, 38, 3098-3100. doi:10.1103/PhysRevA.38.3098

19. Perdew, J. P. Phys. Rev. B 1986, 33, 8822-8824. doi:10.1103/PhysRevB.33.8822

20. Grimme, S. J. Comput. Chem. 2006, 27, 1787-1799. doi:10.1002/jcc.20495

21. Weigend, F.; Ahlrichs, R. Phys. Chem. Chem. Phys. 2005, 7, 3297-3305. doi:10.1039/b508541a

22. Ahlrichs, R.; Bär, M.; Häser, M.; Horn, H.; Kölmel, C. Chem. Phys. Lett. 1989, 162, 165-169. doi:10.1016/0009-2614(89)85118-8

23. Feyereisen, M.; Fitzgerald, G.; Komornicki, A. Chem. Phys. Lett. 1993, 208, 359-363. doi:10.1016/0009-2614(93)87156-W

\section{License and Terms}

This is an Open Access article under the terms of the Creative Commons Attribution License

(http://creativecommons.org/licenses/by/2.0), which permits unrestricted use, distribution, and reproduction in any medium, provided the original work is properly cited.

The license is subject to the Beilstein Journal of Organic Chemistry terms and conditions:

(http://www.beilstein-journals.org/bjoc)

The definitive version of this article is the electronic one which can be found at:

doi:10.3762/bjoc. 9.141 